\title{
PADRÕES DE DISTRIBUIÇÕES ESPACIAIS E TEMPORAIS DE TEMPERATURAS ASSOCIADOS AO BIOMA CERRADO
}

\author{
Patterns of Spatial and Temporal Distributions Temperatures of Surface types associated to \\ Cerrado Biome
}

\author{
Nicali Bleyer Ferreira dos Santos* \\ Laerte Guimarães Ferreira Júnior** \\ Nilson Clementino Ferreira***
}

\begin{abstract}
Resumo
A presença de vegetação é um importante fator na manutenção de processos biogeoquímicos da natureza. No Cerrado, a conversão da vegetação natural em áreas de pastagens e agricultura induz a alteração de variáveis biofísicas como a temperatura de superfície, altamente sensível a mudanças na cobertura da terra. Dessa maneira, com vistas a contribuir com o entendimento da dinâmica de causa e efeito do uso e ocupação do Cerrado nas variáveis biofísicas da região, o presente trabalho procurou analisar, através de dados de sensores orbitais (produto MOD11 - Land Surface Temperature), a correlacão entre a ocupacão da terra e a variabilidade termal de superfície, tendo como recorte temporal o ano de 2003 nos períodos de maio e setembro (início e final da seca). Para tal, essa pesquisa relacionou as diversas formas de ocupação (expressas pelas categorias de uso da terra) com a variável biofísica temperatura de superfície. A análise dos dados mostrou diferenças termais de superfície significativas entre classes naturais e antrópicas, e evidenciou a depedência espacial da variável temperatura de superfície com forte influência da sazonalidade.
\end{abstract}

Palavras-chave: Cerrado, temperatura de superfície, MODIS

\begin{abstract}
The occurrence of vegetation is an important factor regarding the maintainance of natural biogeochemica processes. In the Cerrado, the conversion of the vegetative cover into crop fields and areas of cultivated pastures has induced severe modifications in biophysical variables such as land surface temperatures, highly sensitive to land cover / land use changes. Thus, and aiming at a more thorough understanding of the land conversion impacts on the Cerrado biophysical variables, in this study we assessed, through orbital data (MOD 11 product - Land Surface Temperature), the correlation between land occupation and therma variability, considering 2003 imagery obtained at the beginning and end of the dry season. Data analysis showed significant differences in surface temperature between natural and converted classes and revealed the spatial patterns and dependency of this variable in relation to seasonality.
\end{abstract}

Key words: Cerrado, surface temperature, MODIS

\section{Resumen}

La presencia de vegetación es un factor importante en el mantenimiento de los procesos biogeoquímicos de la naturaleza. En el Cerrado, la conversión de la vegetación natural en las áreas de la agricultura y de pastoreo induce cambios en las variables biofísicas tales como la temperatura de superficie, altamente sensible a los cambios en la cubierta terrestre. Así, con el fin de contribuir a la comprensión de la dinámica de causa y efecto del uso y ocupación de la región del Cerrado en variables biofísicas, este estudio pretende analizar, a partir de informaciones procedentes del sensor orbital (producto MOD11 - Land Surface Temperature), la correlación entre la ocupación de la tierra y la variabilidad térmica de la superfície. El estúdio fue hecho para el año 2003 en los meses de mayo y septiembre (principio y fin de la sequía). Con este fin, su investigación ha vinculado las diversas formas de ocupación (expresadas por las categorías de uso del suelo) con la temperatura de superficie. El análisis de los datos mostro diferencias térmicas significtivas entre las classes naturales e antropizadas y revelo las dependências espaciales de la temperatura de la superfície, com uma fuerte influencia de la estacionalidad.

Motss-clé: Cerrado, temperatura de la superfície, MODIS

(*) Prof ${ }^{\mathrm{a}}$. Drª da Universidade Federal de Goiás, Caixa Postal: 131 CEP 74001-940 Campus Samambaia Goiânia (GO) - Brasil, Tel: (+ 55 62) 35211096 - nicalibleyer@hotmail.com

(**) Prof. Dr. do Instituto de Estudos Sócio-Ambientais da Universidade Federal de Goiás - Campus Samambaia, CEP: 74.001970 - Goiania (GO) - Brasil, Tel: (+55 62) 35211096 - laerte@iesa.ufg.br

(***) Prof. Dr. do Centro Federal de Educação Tecnológica Goiás, Rua 75, número 46, Setor Central, CEP: 74.055110 - Goiania (GO) - Brasil, Tel: (+55 62) 32125050 - ncferreira@uol.com.br 


\section{INTRODUÇÃO}

O Cerrado faz parte do ecossistema das Savanas Tropicais, ambientes fitogeográficos que, em sua maioria, estão situados entre os Trópicos de Câncer e de Capricórnio e são caracterizados pela presença de uma camada contínua de vegetação herbácea e um dossel descontínuo de arbustos e árvores, variando desde campos abertos até formações densas de florestas (GOERT et al, 2008; COSTA e OLSZEVSKI, 2008; RIBEIRO e WALTER, 1998). É o segundo maior bioma do Brasil, ocupando cerca de $23 \%$ do território nacional, e é reconhecido como a savana mais rica do mundo em biodiversidade, com a presença de diversos ecossistemas e riquíssima endemia (MAYERS, 2000) (Figura 1).

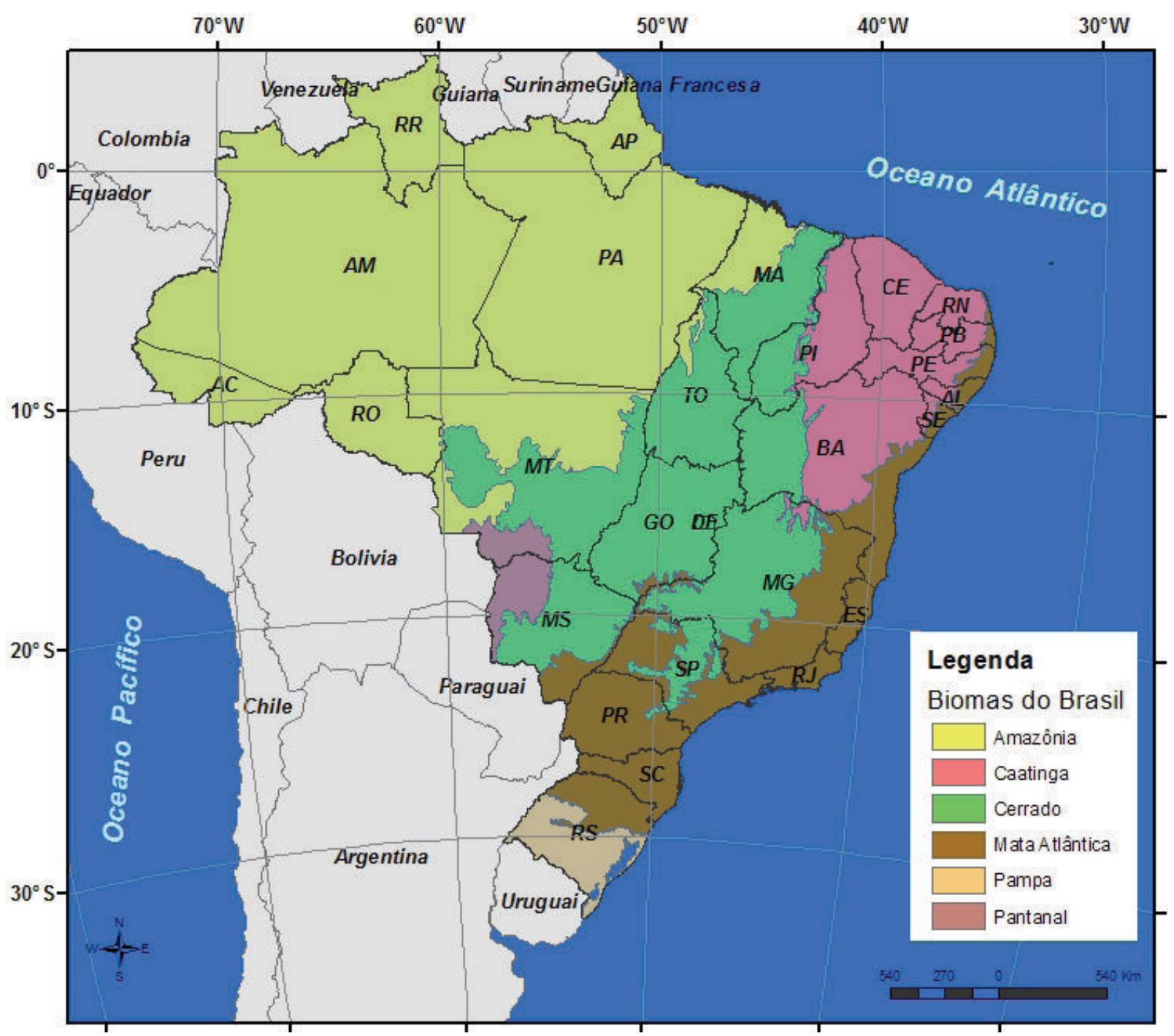

Figura 1 - Mapa de localização do Cerrado e demais biomas do Brasil. Fonte: Ministério do Meio Ambiente e Instituto Brasileiro de Geografia e Estatística - IBGE, 2005/elaboração própria.

No contexto nacional, o valor ecológico do Cerrado se destaca, dentre outros fatores, pela importância que exerce na manutenção de importantes bacias hidrográficas brasileiras, como as dos rios Paraná, São Francisco, Araguaia e Tocantins, bem como no papel de sumidouro dos gases de efeito estufa. Além disso, a consagração como área de expansão agropecuária, incentivada por políticas territoriais de ocupação, conferem ao bioma, posição de destaque no cenário econômico brasileiro, principalmente, no que diz respeito à produção de grãos e pecuária extensiva para exportação (RATTER et al, 1996). 
Apesar de tamanha importância, cerca de $40 \%$ de sua área já foi convertida em pastagens, áreas agrícolas e urbanas (SANO, et al 2008), fazendo com que a taxa de conversão para a agricultura e a pecuária seja da ordem de $12.480 \mathrm{~km}^{2}$ por ano (MARTINS JR \& CHAVES, F., 2009). Fatores como a interiorização da capital federal e o decorrente processo de expansão da infra-estrutura, muito contribuíram para a conversão das áreas de vegetação nativa, o que certamente induziu a alterações nos parâmetros biofísicos da região, uma vez que a presença de vegetação é um importante fator na regulação de processos biogeoquímicos (como os ciclos da água e de energia) e na manutenção das flutuações sazonais do clima (CHUDNOVSKY et al, 2004; BERBET e COSTA, 2003; COSTA e FOLEY, 1997; NOBRE et al, 1991).

Nesse contexto, um parâmetro a ser considerado é a temperatura de superfície, isso porque essa variável é um importante fator na estimativa do balanço de energia da superfície e altamente susceptível ao padrão de ocupação da terra (HASHIMOTO et al, 2003). Devido à escassez e dificuldade em se coletar dados meteorológicos em campo, a medição da temperatura de superfície tem contado com o auxílio de importantes avanços tecnológicos para sua mesuração, como por exemplo, os dados coletados através de sensores orbitais. Tal fato se justifica devido aos avanços recentes na área de sensoriamento remoto, com a possibilidade de obtenção de dados em resolução temporal e espacial variada (ANDERSEN, 1997; SALISBURY and D' ARIA, 1992), bem como a proximidade entre os dados medidos in loco e os dados estimados pelo sensor (WAN, 2007a).

Nesse sentido, o presente trabalho procurou fornecer subsídios que possam contribuir com o entendimento do comportamento termal de superfície bem como a dinâmica de causa e efeito do uso e ocupação do Cerrado nas varáveis biofísicas. Para tal, essa pesquisa relaciona as diversas formas de uso e ocupação com a variável biofísica temperatura de superfície, assim como avalia a influência de fatores externos como a latitude, a altitude e a sazonalidade na variabilidade termal de superfície.

\section{MATERIAIS E MÉTODOS}

O presente estudo abrange toda a área do Cerrado, aproximadamente 2.046 milhões de $\mathrm{km} 2 \mathrm{e}$ foi desenvolvido a partir de informações obtidas pelo sensor MODIS (Moderate Resolution Imaging Spectroradiometer). A escolha pela área é explicada, dentre outros fatores, pela grande importância do bioma no cenário da biodiversidade mundial - hotspots (MYERS et al, 2000) e à rápida conversão de suas paisagens naturais (SANO, 2008; SANO 2009). A Figura 2 mostra a localização da área de estudo com as cenas (tiles) do sensor MODIS utilizadas na pesquisa.

As informações utilizadas para a análise de temperatura de superfície - Land Surface Temperature (LST) são provenientes do produto MOD11 A2, Coleção 5, com resolução espacial de $1 \mathrm{~km}$. O MOD11 utiliza o algoritmo LST (Land Surface Temperture) para o cálculo de temperatura de superfície, incluindo o Day/night LST algorithm (WAN and LI, 1997), desenvolvido especificamente para o MODIS, que produz imagens termais diurnas e noturnas para toda a superfície da Terra, com periodicidade diária. O produto MOD11 A2 foi escolhido por ser uma composição de oito dias gerados a partir de dados do MOD11 A1 (dados diários) permitindo a aquisição de dados que, por algum motivo, tiveram sua determinação prejudicada em função, principalmente, de interferências atmosféricas. A coleção 5 (V5) foi utilizada em lugar da coleção 4 (V4), por apresentar melhorias metodológicas significativas, proporcionando dados de maior acurácia (WAN, 2007b).

Os dados provenientes do produto MOD11 A2 foram filtrados com o auxílio do Controle Automático de Qualidade (Quality Control - QC), que acompanha cada imagem LST, e é fornecido para cada pixel (WAN et al, 1998; WAN, 2007b). 


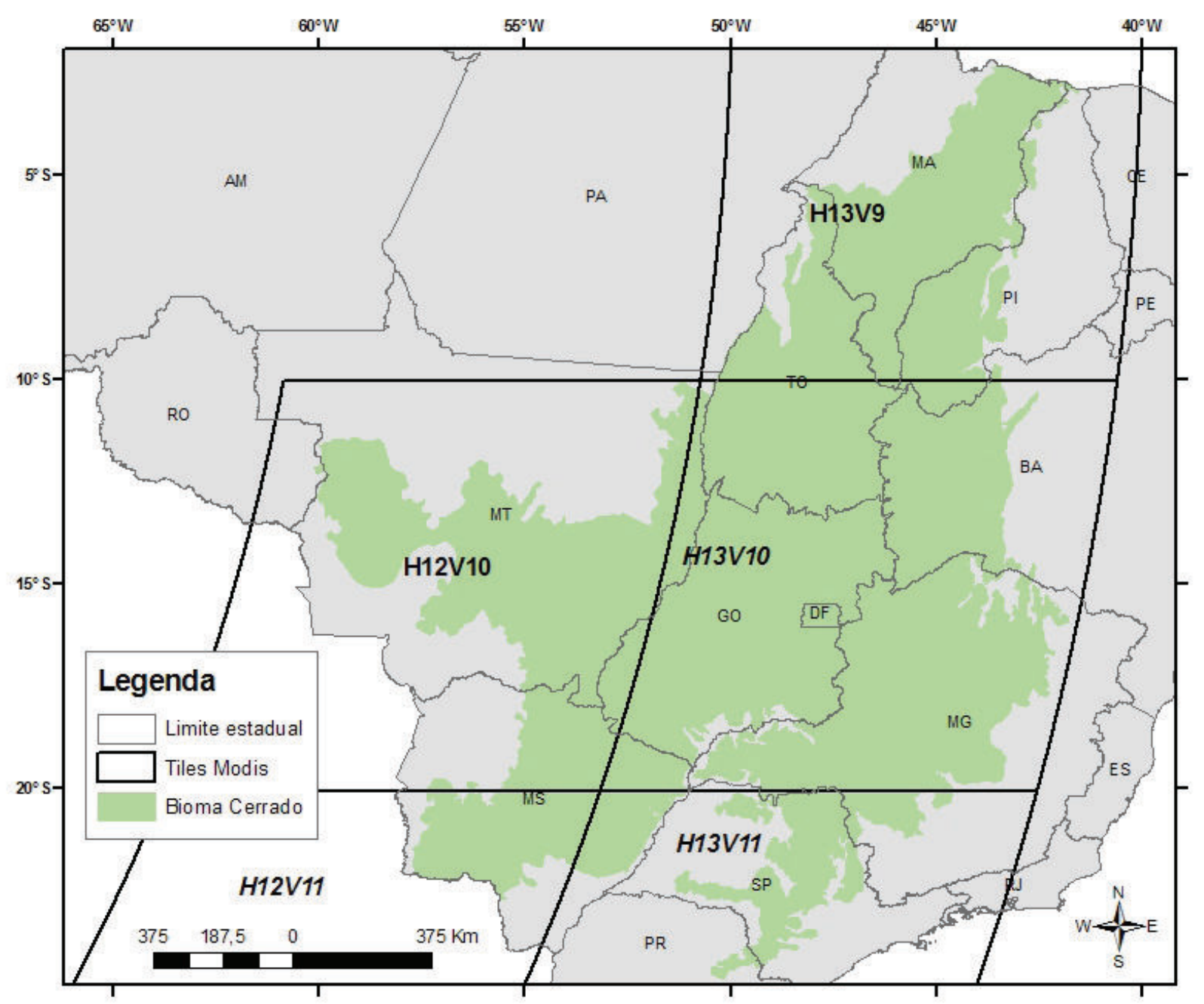

Figura 2: Mapa de localização da área de estudo e dos tiles MODIS utilizados na pesquisa. Elaboração própria.

Dentre as duas plataformas disponíveis para a obtenção dos dados de temperatura de superfície (TERRA e AQUA), optou-se pela utilização do satélite TERRA, visto que análises preliminares mostraram que as informações obtidas através dessa plataforma apresentam menor contaminação das informações por nuvens, após a aplicação do Quality Control (Figura 3).

As análises foram feitas a partir de um recorte sazonal, utilizando as imagens referentes aos meses de maio e de setembro (dia 129 e 257), devido à importância que a sazonalidade exerce no bioma, tanto no que se refere às condições biofísicas, quanto à forte correlação temporal e espacial da vegetação com a ocorrência de chuvas (RATANA et al, 2005; FERREIRA E HUETE, 2004; FERREIRA et al, 2003).

Os dados de LST foram então corelacionados com os tipos de uso e ocupação da terra no bioma Cerrado, delimitados com base no mapeamento do Projeto de Conservação e Utilização Sustentável da Diversidade Biológica Brasileira - PROBIO, elaborado em 2002, na escala 1:250.000, considerando dez (10) classes de maior representatividade espacial no bioma, sendo cinco (5) classes com influência antrópica (agricultura, pastagem, mineração, área urbana e reflorestamento) e cinco (5) classes consideradas como vegetação remanescente (savana estépica, savana florestada, savana gramíneo-lenhosa, savana arborizada e savana parque). A Figura 4 mostra a localização das classes utilizadas na pesquisa. 


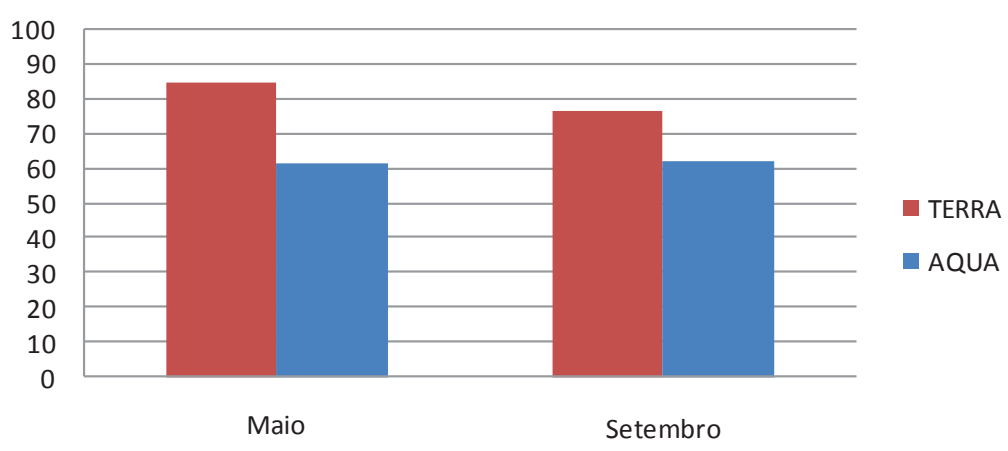

Figura 3 - Gráfico de comparação dos dados LST obtidos pela plataforma TERRA e pela plataforma AQUA após a aplicação do controle automático de qualidade, para o mês de maio e setembro. Elaboração própria.

As classes foram amostradas para cada grau de latitude sendo que, para cada amostra, obteve-se um total de 9 pixels. Com base nas coordenadas geográficas, os dados de temperatura de superfície foram correlacionados com as respectivas latitudes médias, bem como com os dados de altitude, obtidos a partir de imagens do Shuttle Radar Topography Mission SRTM (Figura 5).

Para cada grupo de amostra foram calculados a média, o desvio padrão e o coeficiente de variação, assim como a distribuição da frequência de temperatura das diversas classes de uso e ocupação. Este último procedimento foi realizado considernado as classes de vegetação remanescente em dois grupos: cerrado denso e cerrado ralo, com objetivo de fascilitar as comparações entre as diferentes classes.

Tendo em vista que as variáveis biofísicas como a temperatura de superfície, bem como a grande maioria dos fenômenos ecológicos, estão sujeitos a autocorrelação espacial (LEGENDRE, 1993), foi alculado, para os dois períodos de análise (i.e. DOY 129 e 257), o Índice de Moran. Esse índice, que varia de -1 a 1 , mede o grau de dependência espacial dos dados através da similaridade entre observações de pares de localidades para cada classe de distância (Valores positivos de I de Moran indicam autocorrelação positiva, i.e., células espacialmente mais próximas são mais similares) (CARVALHO et al, 2008). Especificamente, o comportamento da autocorrelação foi avaliado para uma distância de até $50 \mathrm{~km}$, conforme um intervalo de $1 \mathrm{~km}$ e uma vizinhança definida por quatro localidades adjacentes à cada pixel.

As diferenças entre as médias de temperatura, conforme os dois períodos investigados (i.e. DOY 129 e 257) foi avaliada através do teste de Tukey, o qual, com base na diferença mínima significativa (DMS) identifica se, no intervalo espacial analisado, se há diferenças significativas ( $\mathrm{p}$ $<0.05)$ entre as médias amostradas das diferentes classes.

\section{RESULTADOS E DISCUSSÕES}

Como se pode observar nas figuras de 7 a 13, os valores de temperatura média amostrados em cada classe apresentam nítida diferença sazonal entre os valores de temperatura registrados em maio (cor azul do gráfico) e em setembro (cor vermelha da figura 6). Em praticamente todas as classes (com exceção da savana estépica), o intervalo no qual se concentrou a maior parte das médias de temperatura amostradas foi o de $25^{\circ} \mathrm{C}$ e $30^{\circ} \mathrm{C}$, no mês de maio, e de $35^{\circ} \mathrm{C}$ e $45^{\circ} \mathrm{C}$, no mês de setembro. Apesar de esperado que valores de temperatura sejam intrinsecamente relacionados com a localização geográfica (maiores latitudes, menores valores de temperatura), as informações presentes nos gráficos não evidenciaram uma relação acentuada entre a variabilidade termal e a latitude. Apenas em algumas classes, como o reflorestamento, a savana florestada, a savana estépica e a savana gramíneo lenhosa, observam-se uma leve tendência de queda de temperatura nas latitudes a partir de $-11^{\circ}$. E, na agricultura e no reflorestamento, um pequeno indicativo de temperaturas mais elevadas nas amostras localizadas em latitudes menores. 

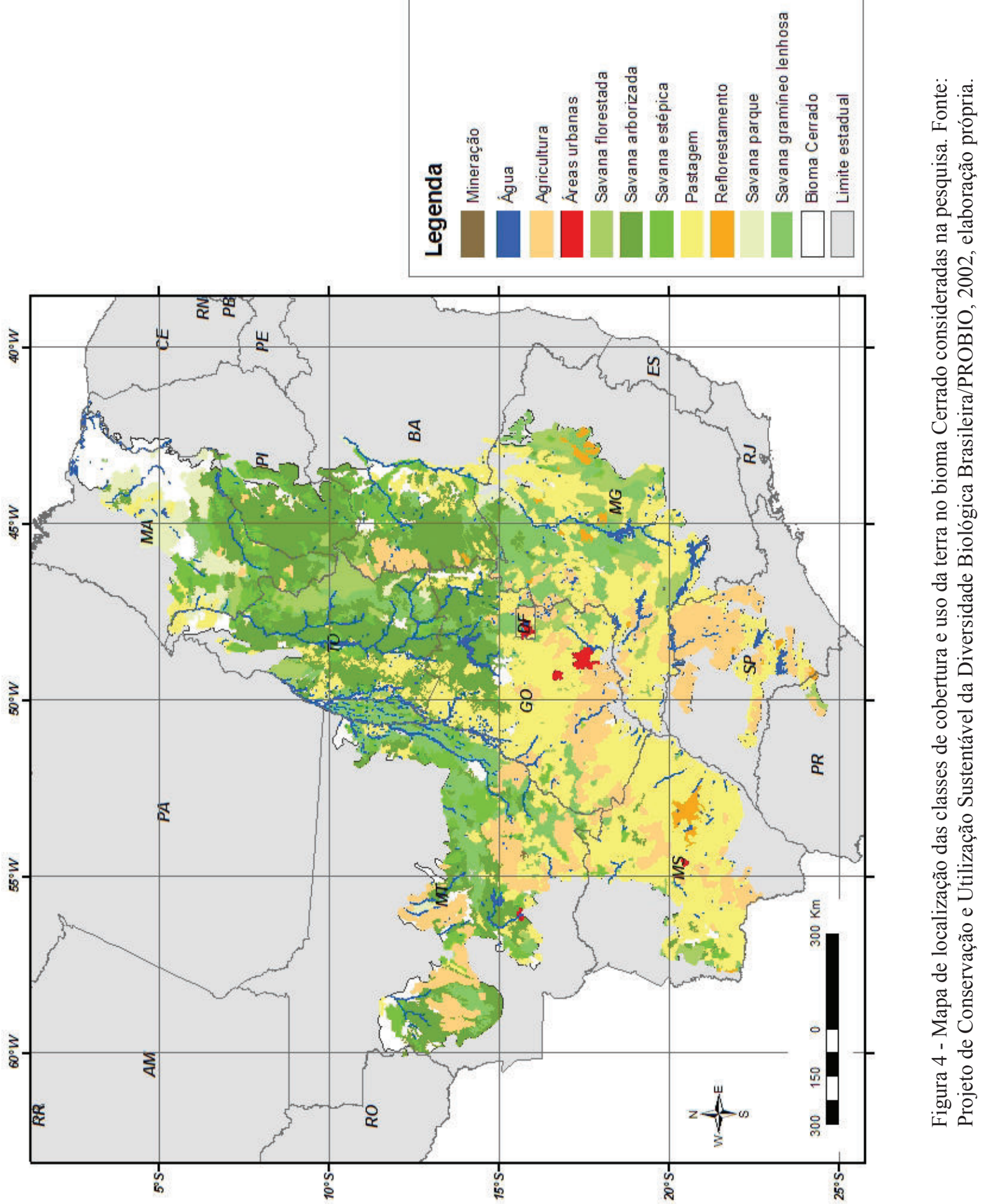


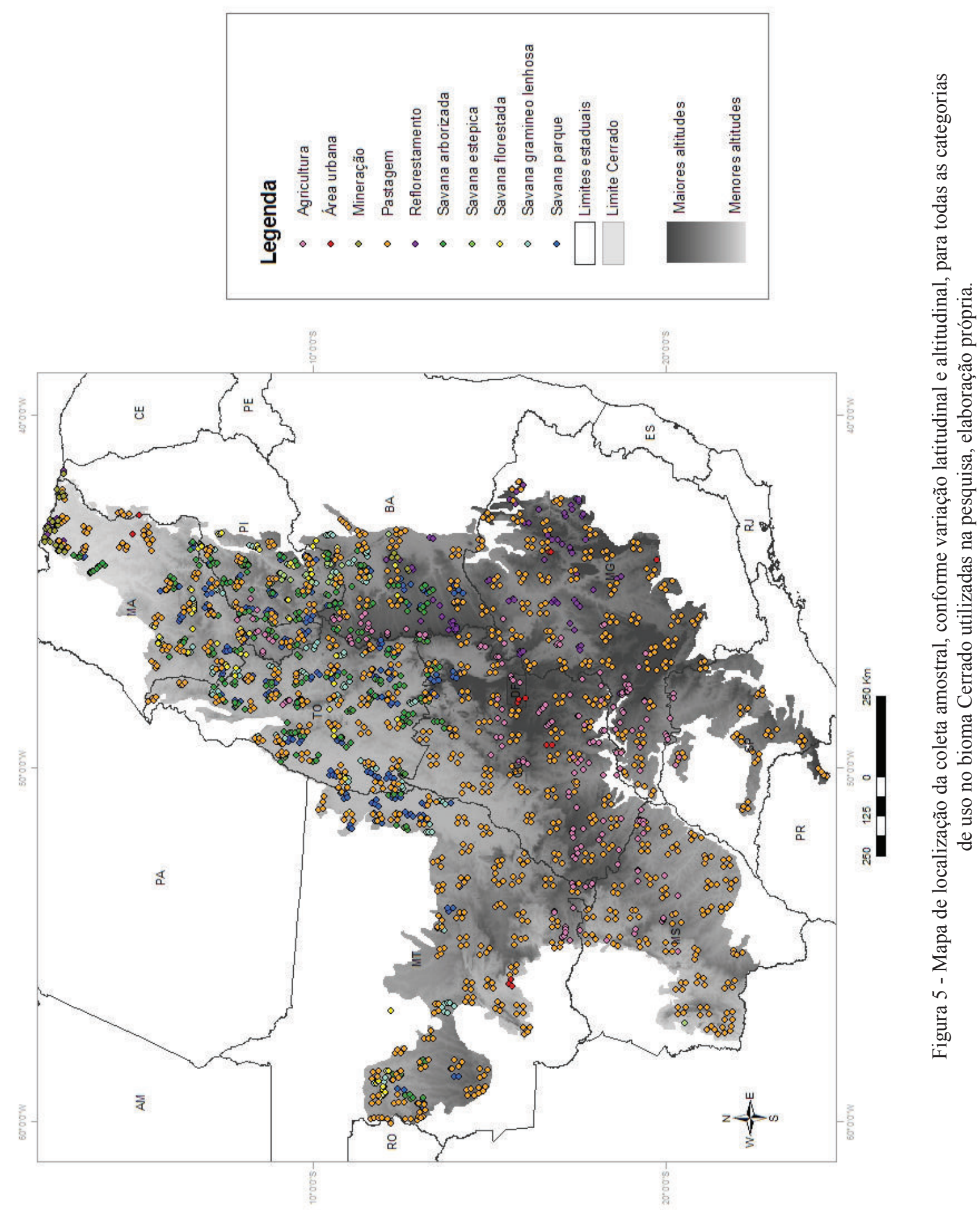




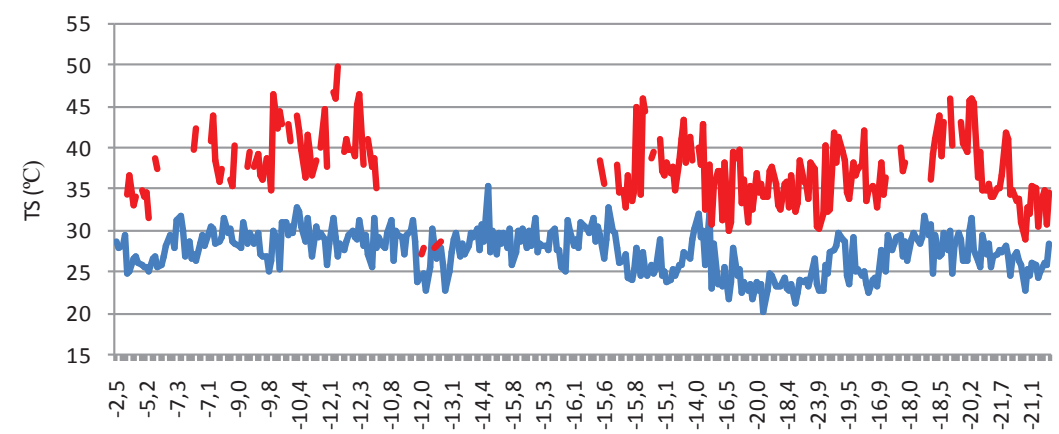

Figura 6 - Gráfico das distribuições das médias termais de superfície para a classes pastagem, conforme distribuição latitudinal. A cor azul representa a temperatura no mês de maio e a cor vermelha a temperatura no mês de setembro.

Elaboração própria.
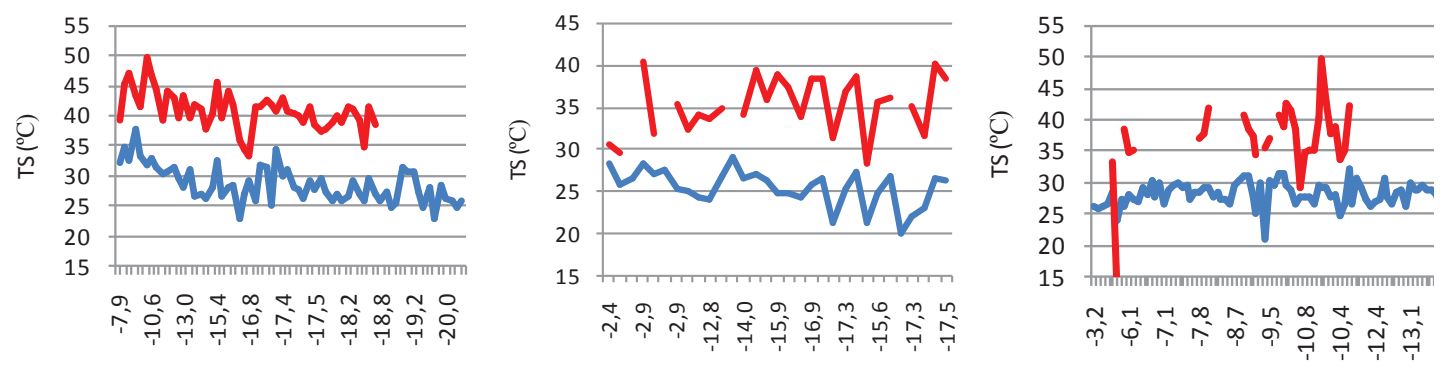

Figuras 7, 8 e 9 - Gráficos da istribuição das médias termais de superfície para as classes agricultura, reflorestamento e savana arborizada (respectivamente), conforme distribuição latitudinal. A cor azul representa a temperatura no mês de maio e a cor vermelha a temperatura no mês de setembro. Elaboração própria.
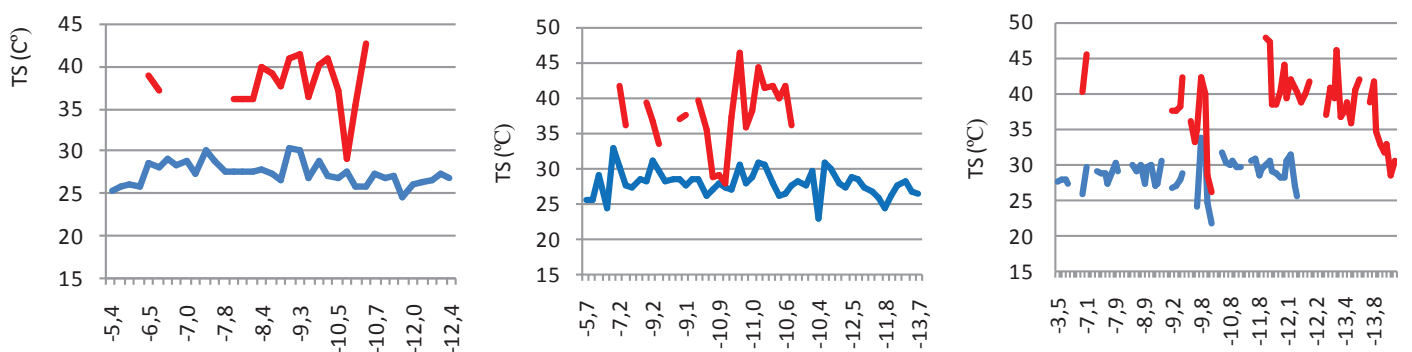

Figuras 10, 11 e 12: Distribuição das médias termais de superfície para as classes savana florestada, savana gramíneo lenhosa e savana parque (respectivamente), conforme distribuição latitudinal. A cor azul representa a temperatura no mês de maio e a cor vermelha a temperatura no mês de setembro. Elaboração própria.

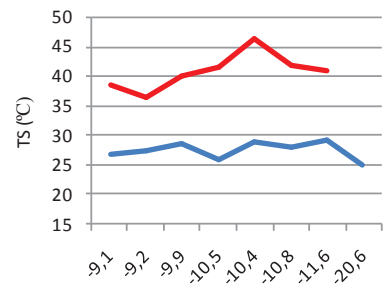

Figura 13 - Gráfico da Distribuição das médias termais de superfície para a classe savana estépica conforme distribuição latitudinal. A cor azul representa a temperatura no mês de maio e a cor vermelha a temperatura no mês de setembro. Elaboração própria. 
A análise dos coeficientes de ajuste (r2) corrobora a relativa dependência entre a temperatura média da savana estépica e a latitude (para ambas as épocas do ano), bem como evidencia uma dependência latitudinal das classes agricultura e reflorestamento, principalmente em maio (Tabela 1), em decorrência do caráter bastante localizado destas duas classes antrópicas no bioma.

Tabela 1: Correlação entre as médias de temperatura de superfície e a latitude para diferentes classes de uso e cobertura da terra no bioma Cerrado: valores de $\mathrm{R}^{2}$ para LST e latitude para o ano de 2003

\begin{tabular}{l|r|r}
\hline CLASSES DE USO E COBERTURA DA TERRA & \multicolumn{1}{l|}{ Maio } & \multicolumn{1}{l}{ Setembro } \\
\hline Agricultura & 0,42 & 0,29 \\
\hline Pastagem & 0,13 & 0,06 \\
\hline Reflorestamento & 0,21 & 0,08 \\
\hline Savana Arborizada & 0,00 & 0,12 \\
\hline Savana Estépica & 0,33 & 0,32 \\
\hline Savana Florestada & 0,05 & 0,00 \\
\hline Savana Gramíneo Lenhosa & 0,00 & 0,02 \\
\hline Savana Parque & 0,00 & 0,00 \\
\hline
\end{tabular}

No que diz respeito às altitudes, é interessante observar (Tabela 2) que as maiores dependências ocorrem para as temperaturas médias das classes antropizadas, i.e. reflorestamento, pastagem e agricultura, com valores de r 2 de $0.22,0.18$ e 0.14 , respectivamente. Essa dependência pode ser parcialmente explicada pela "padronização" dos locais em que normalmente essas classes são encontradas: relevos planos, com pouca variação de altitude. Por outro lado, a maior correlação (r2 $=0.32$ ) ocorre para a classe savana estépica. Tanto para as classes antrópicas, quanto para a classe de vegtação nativa, é também interessante observar, que as maiores correlações ocorrem em maio à semelhança do que foi observado quanto às latitudes.

Tabela 2: Correlação entre os dados de temperatura de superfície e altitude para diferentes classes de uso e cobertura da terra no bioma Cerrado: valores de $\mathrm{R}^{2}$ para LST e altitude para o ano de 2003

\begin{tabular}{l|r|r}
\multicolumn{1}{c|}{ CLASSES DE USO } & \multicolumn{1}{c|}{ Maio } & \multicolumn{1}{c}{ Setembro } \\
\hline Agricultura & 0,14 & 0,10 \\
\hline Pastagem & 0,18 & 0,01 \\
\hline Reflorestamento & 0,22 & 0,04 \\
\hline Savana Arborizada & 0,00 & 0,16 \\
\hline Savana Estépica & 0,37 & 0,13 \\
\hline Savana Florestada & 0,00 & 0,08 \\
\hline Savana Gramíneo Lenhosa & 0,00 & 0,08 \\
\hline Savana Parque & 0,05 & 0,04 \\
\hline
\end{tabular}

A análise de coeficiente de variação (CV) mostrou valores baixos, o que corrobora certa homogeneidade nos conjuntos de dados amostrados. Os "maiores" valores de CV registrados tanto no mês de maio, quanto no mês de setembro $(0,02$ a 0,06$)$, foram referentes às classes reflorestamento e agricultura. Este fato pode ser parcialmente explicado pela heterogeneidade das culturas agrícolas e dos reflorestamentos, representada pelos diversos estágios de crescimento, assim como a diversidade de espécies de plantas que são utilizadas tanto nos reflorestamentos quanto na agricultura. Já os menores valores de CV foram verificados na savana parque e na savana arborizada, seguidos da savana gramíneo lenhosa, da savana estépica e da pastagem. Nessas classes, o conjunto de características fitofisionômicas (a variedade de espécies, o tamanho e a dispersão espacial de seus indivíduos) contribui para uma "homogeneização" da paisagem, principalmente nos locais aonde foram coletadas as amostras, "padronizando" as temperaturas de superfície (Figuras de 14 a 21). 


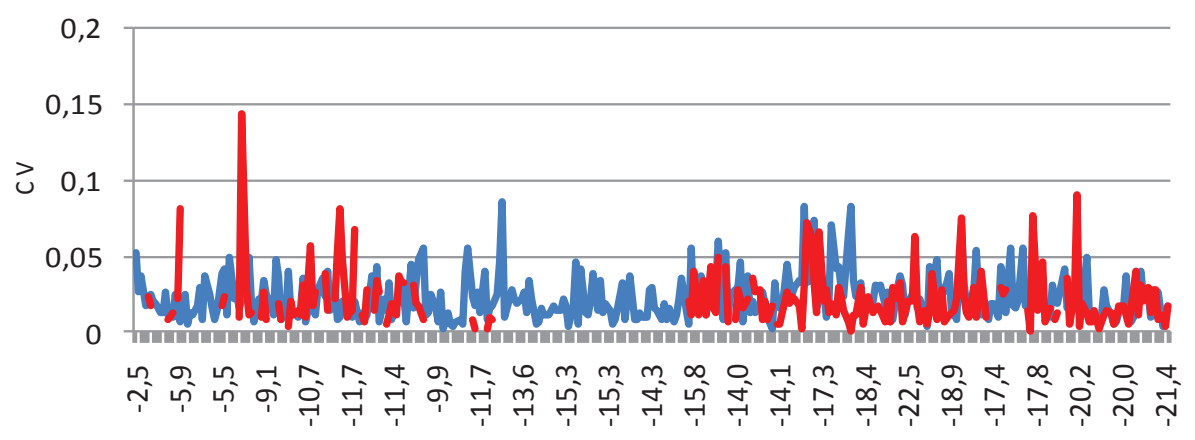

Figura 14 - Gráfico da distribuição do coeficiente de variação para a pastagem conforme distribuição latitudinal. A cor azul representa o coeficiente de variação no mês de maio e a cor vermelha o coeficiente de variação no mês de setembro. Elaboração própria.
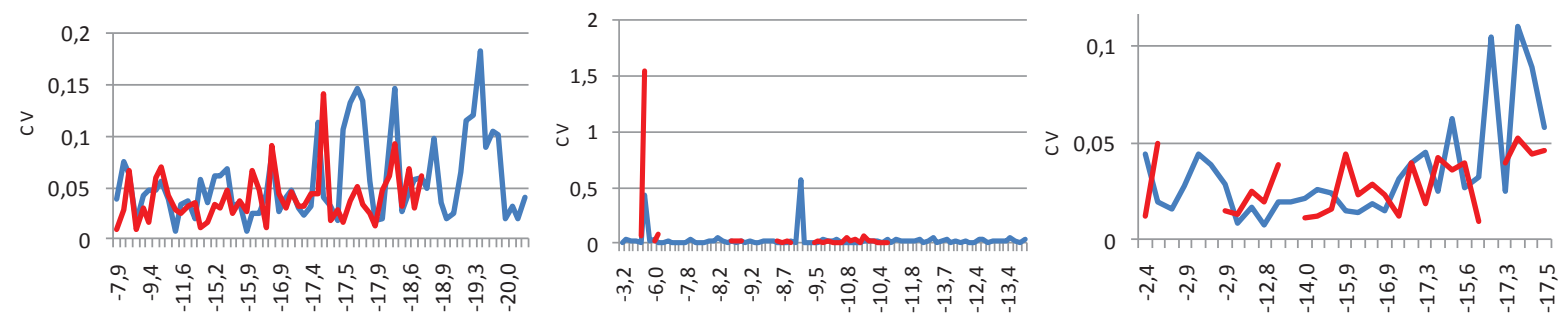

Figuras 15, 16 e 17 - Gráficos da Distribuição do coficiente de variação para as classes agricultura, reflorestamento e savana arborizada (respectivamente), conforme distribuição latitudinal. A cor azul representa o coeficiente de variação no mês de maio e a cor vermelha o coeficinte de variação no mês de setembro. Elaboração própria.
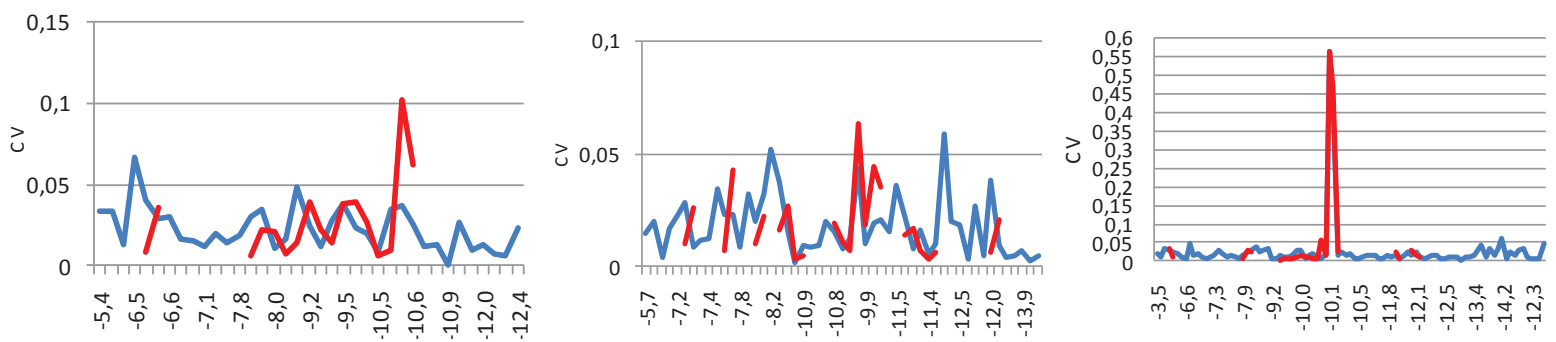

Figuras 18, 19 e 20 - Gráficos da distribuição do coeficiente de variação para as classes savana florestada, savana gramíneo lenhosa e savana parque (respectivamente), conforme distribuição latitudinal. A cor azul representa as temperaturas no mês de maio e a cor vermelha as temperaturas no mês de setembro. Elaboração própria.

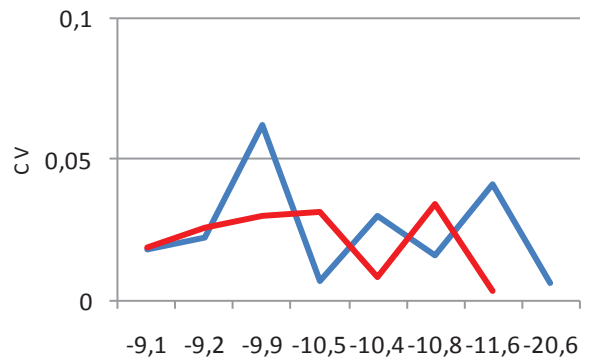

Figura 21 - Gráficos do coeficiente de variação para a classe savana estépica conforme distribuição latitudinal. A cor azul representa as temperaturas no mês de maio e a cor vermelha as temperaturas no mês de setembro. Elaboração própria. 
É interessante observar os amplos intervalos de temperatura, entre maio e setembro, e as sobreposições destes intervalos entre as várias classes consideradas. A disparidade observada reforça a interferência da sazonalidade na temperatura de superfície das classes de uso e ocupação do Cerrado (Figura 22 e 23).
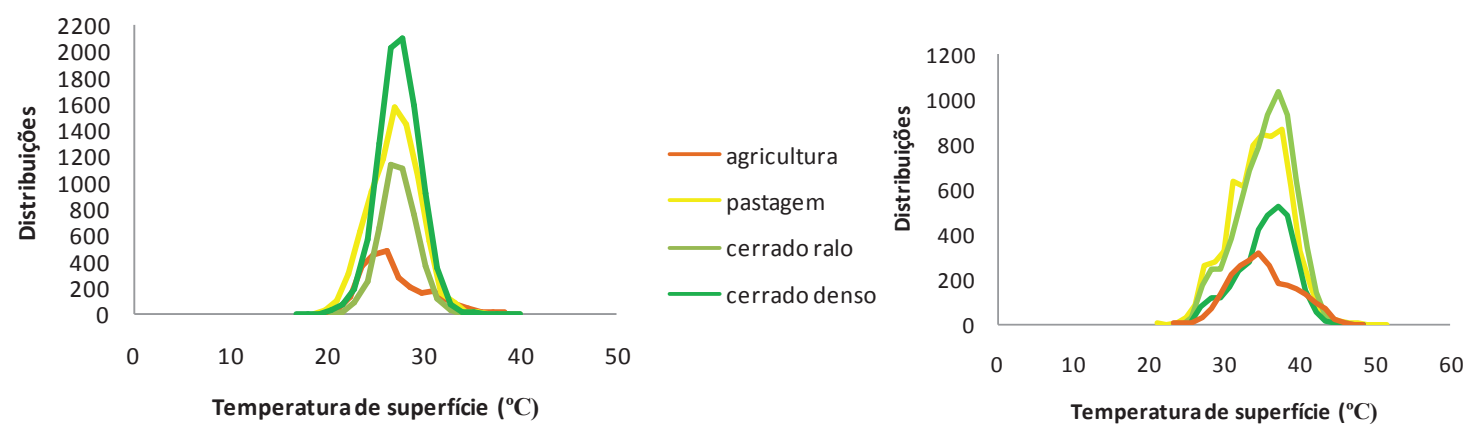

Figuras 22 e 23 - Gráficos das distribuições de frequência (temperatura de superfície) das classes de uso e cobertura da terra no Cerrado para os meses de maio e setembro, respectivamente. Elaboração própria.

Conforme observado, a temperatura da superfície é resultado de uma combinação complexa de fatores tanto intrínsecos (tipos de solos e coberturas, substrato rochoso, etc), quanto extrínsecos (topografia, iluminação solar, proximidade entre alvos, etc), os quais resultam, invariavelmente, em homogeneização das respostas entre alvos e conseqüentemente dependência espacial. O correlograma com os valores do índice de Moran (Figura 24), cujos valores são significativos até a distância de $50 \mathrm{~km}$, confirma que a temperatura de superfície não só apresenta uma grande dependência espacial, como também é altamente estruturada espacialmente. Ou seja, boa parte da variância termal que observamos, além de ocorrer em função do tipo de cobertura e substrato, ocorre também pelo efeito da variabilidade espacial em si. Por outro lado, a menor correlação espacial observada em setembro, quando esperamos mais diversidade na superfície (comparativamente a maio, quando os alvos vegetados estão invariavelmente "verdes"), mais uma vez corrobora o papel fundamental desempenhado pelos vários tipos de cobertura quanto à distribuição e variação dos valores de temperatura da superfície.

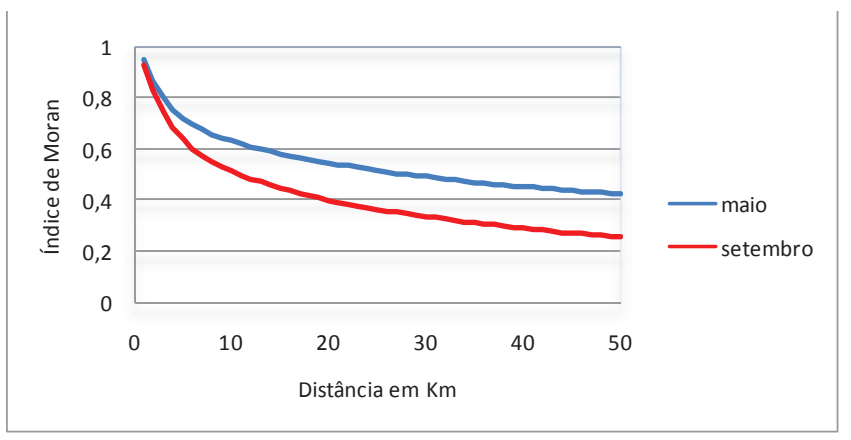

Figura 24 - Gráfico da distribuição dos valores referentes à dependência espacial (Índice de Moran) dos dados de temperatura de superfície no bioma Cerrado para maio e setembro. Elaboração própria.

A importância da sazonalidade na variação dos dados de temperatura, bem como o seu efeito distinto sobre cada classe de cobertura e uso da terra observada nas análises anteriores, é reiterada na figura 25 , na qual, cinco médias de temperatura para cada classe, escolhidas aleatoriamente, são representadas no espaço sazonal definido pelas datas maio e setembro. Conforme a distribuição das amostras entre as isolinhas de temperatura, percebe-se que as variações concentram-se entre 0 e $70 \%$. Especificamente em relação à classe pastagem, é interessante observar que esta apresenta um 
amplo intervalo de variação sazonal, o qual se estende aproximadamente de 0 a $80 \%$. No âmbito das classes naturais, destacam-se as classes de savana estépica e de savana arborizada, com variações próximas à 70 e $35 \%$, respectivamente.
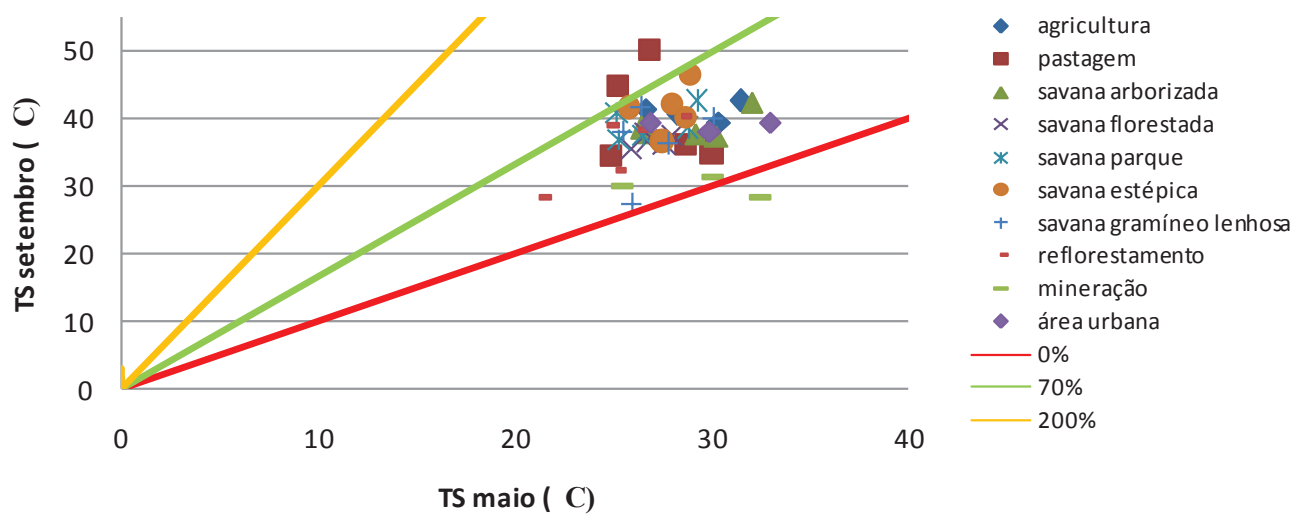

TS maio ( C)

Figura 25 - Gráfico do comportamento sazonal da temperatura de superfície para as principais classes de cobertura e uso da terra no bioma Cerrado. Elaboração própria.

Especificamente no que diz respeito às diferenças entre as médias de temperatura, entre as várias classes de cobertura e uso da terra, estas foram avaliadas através do teste de Tukey. Este teste, que tem como base a diferença mínima significativa entre os pares das médias amostrais, foi calculado também consideradno as classes mais reprsentativas espacialmente, considerando as diferentes classes de savana como uma categoria única, para fascilitar a comparação com o valor das médias termais da pastagem, da agricultura e do reflorestamento. Assim, valores de p menores do que 0,05 indicam que as médias entre as classes são estatisticamente distintas. Como observado na tabela 3, para um intervalo de confiança de $95 \%$, os resultados mostraram que no mês de maio, as médias termais de superfície só não se diferenciaram entre as classes agricultura e savana. Já no mês de setembro, as diferenças das médias termais entre as classes, em sua maioria, não foram significativas, diferenciando-se apenas quando comparadas a agricultura.

Tabela 3: Teste de Tukey - diferença mínima entre os pares das médias amostrais de temperatura de superfície, em maio e setembro, para as classes de cobertura e uso do solo no bioma Cerrado.

\begin{tabular}{|c|c|c|c|c|c|c|c|c|c|}
\hline \multirow[t]{2}{*}{ CLASSE } & \multirow[t]{2}{*}{ Classe } & \multirow[t]{2}{*}{ Diferença } & \multirow{2}{*}{$\begin{array}{l}\text { Valor-p } \\
\text { (Maio) }\end{array}$} & \multicolumn{2}{|c|}{$\begin{array}{l}\text { 95\% Intervalo de } \\
\text { confiança }\end{array}$} & \multirow[t]{2}{*}{ Diferença } & \multirow{2}{*}{$\begin{array}{l}\text { Valor-p } \\
\text { (setembro) }\end{array}$} & \multicolumn{2}{|c|}{$\begin{array}{l}95 \% \text { Intervalo de } \\
\text { confiança }\end{array}$} \\
\hline & & & & inferior & superior & & & inferior & superior \\
\hline agricultura & pastagem & 1.209 & 0.001 & 0.394 & 2.024 & 3.589 & 0 & 1.897 & 5.28 \\
\hline agricultura & Reflorestamento & 3.143 & 0 & 1.834 & 4.452 & 5.667 & 0 & 3.117 & 8.217 \\
\hline agricultura & Savanna & 0.521 & 0.37 & -0.308 & 1.35 & 3.098 & 0 & 1.303 & 4.892 \\
\hline pastagem & Reflorestamento & 1.934 & 0 & 0.811 & 3.056 & 2.078 & 0.065 & -0.086 & 4.242 \\
\hline pastagem & Savanna & -0.688 & 0.001 & -1.171 & -0.205 & -0.491 & 0.71 & -1.674 & 0.691 \\
\hline reflorestamento & Savanna & -2.622 & 0 & -3.754 & -1.49 & -2.569 & 0.017 & -4.814 & -0.324 \\
\hline
\end{tabular}

Ainda que no conjunto total de dados, predominem as diferenças significativas, a baixa separabilidade observada entre algumas classes, principalmente no mês de setembro, pode ser atribuída à elevada autocorrelação espacial envolvendo os dados de temperatura, bem como à grande variabilidade interna acentuada durante o período seco. 


\section{CONSIDERAÇÕES FINAIS}

O produto MOD11 A2, proveniente do sensor MODIS, mostrou-se adequado ao monitoramento da temperatura de superfície em âmbito regional, confirmando a possibilidade de uso de sensores orbitais (com cobertura espacial e temporal variada), como instrumentos auxiliares na coleta de dados termais. Apesar de ser um sensor de média resolução espacial, em que a presença de vários tipos de cobertura em um mesmo pixel dificulta a captação da temperatura real de cada alvo, os dados de Land Surface Temperature foram capazes de distinguir o comportamento termal dos diferentes tipos de uso e cobertura da terra no Cerrado, evidenciando, inclusive, o comportamento sazonal dessas classes.

Ainda que algumas classes apresentassem certa dependência da temperatura de superfície em relação às variáveis latitude e altitude, tal fato não se mostrou tão forte no contexto geográfico do Cerrado, o que pode ser parcialmente explicado por não haver mudanças latitudinais e altitudinais drásticas no bioma. Além disso, evidencia que outros elementos tanto intrínsecos quanto extrínsecos à superfície, estão atuando e contribuindo para que ocorram as diversidades termais. Dentre os fatores extrínsecos, a sazonalidade mostrou-se um fator determinante na resposta termal, com efeitos distintos em cada classe de uso e cobertura da terra.

Apesar de no conjunto total de dados ter ocorrido a predominância de diferenças termais significativas entre classes antrópicas e classes de vegetação remanescente, a avaliação da variável temperatura de superfície mostrou-se altamente complexa. A autocorrelação espacial dessa variável, somada à grande variabilidade de temperatura interna a cada classe, acentuadas pela sazonalidade do bioma, são alguns dos fatores que dificultam o mapeamento da TS em nível regional.

Dessa forma, sendo a temperatura de superfície uma variável tão importante no equilíbrio do sistema superfície-atmosfera e tão complexa de ser exatamente mensurada, algumas recomendações tanto de cunho técnico quanto político, que visam melhorar o nível de entendimento/detalhamento do comportamento dessa variável no bioma Cerrado, precisam ser levadas em consideração.

As recomendações técnicas que, consideramos de suma importância são:

1. Como no bioma Cerrado há uma grande variedade de usos e cobertura da terra que se (re) estabelecem em uma diversidade de estágios e ciclos de crescimentos, a temperatura de superfície deve ser avaliada ao longo de um ciclo anual, de modo que os dados evidenciem seu comportamento cíclico e não apenas sazonal. Dessa forma, em trabalhos futuros é necessário realizar ánalises da TS que considerem todos os meses do ano.

2. O comportamento termal de superfície das diversas classes de uso e ocupação da terra no bioma Cerrado deve ser confirmado através de um maior período de análise. Portanto, se faz necessário um estudo a partir de séries temporais.

3. Como a variabilidade termal de superfície está fortemente relacionada com o tipo de uso e cobertura do solo e como sensores de média resolução espacial, devido ao tamanho de seus pixels, não são capazes de exprimir com exatidão a temperatura dos diversos alvos, torna-se necessário análises com maior refinamento dos dados, considerando áreas específicas, a partir de sensores termais de maior resolução espacial.

4. É importante quantificar a influência da topografia na temperatura de superfície, bem como a influência de fatores como o tipo de solo e a quantidade de água presente no substrato, a partir de medições locais em áreas onde seja possível o isolamento desses fatores nas diferentes classes de uso.

5. A correlação entre a temperatura do ar e a temperatura de superfície no bioma Cerrado pode ser explorada a partir de estudos que comparem os dados estimados pelo sensor MODIS, com os dados mensurados em campo. Como existem poucas estações meteorológicas em pleno 
funcionamento no bioma, o estudo poderia ser desenvolvido a partir de métodos de extrapolação dos dados absolutos. Essas análises seriam importantes para verificar a possibilidade de utilizar dados orbitais para a confecção de modelos de balanço de energia em âmbito regional.

\section{AGRADECIMENTOS}

Este trabalho, desenvolvido no âmbito do Programa Multidisciplinar de Doutorado em Ciências Ambientais da Universidade Federal de Goiás (CIAMB / UFG), se insere entre as várias iniciativas do Laboratório de Processamento de Imagens e Geoprocessamento (LAPIG / UFG) voltadas ao monitoramento sistemático e gestão territorial do bioma Cerrado. O segundo e terceiro autores são bolsistas de Produtividade em Pesquisa (1C) do Conselho Nacional de Desenvolvimento Científico e Tecnológico $(\mathrm{CNPq})$.

\section{REFERÊNCIA BIBLIOGRÁFICA}

ANDERSEN, H.S, Land surface temperature estimation based on NOAA-AVHRR data during the HAPEX-Sahel experiment. Journal of Hydrology, v.189, p.788-814. 1997.

BERBET, M. L. C. ; COSTA, M. H. . Climate change after tropical deforestation: seasonal variability of surface albedo and its effects on precipitation change. Journal of Climate, Boston, v. 16, n. 12, p. 20992104, 2003.

CARVALHO de, V. M. F.; FERREIRA, G. L.; LOBO, C. F.; DINIZ, F. A. Padrões de autocorrelação espacial de índices de vegtação MODIS no bioma Cerrado. Revista Árvore, Viçosa-MG, v.32, n.2, p.279-290, 2008. CHUDNOVSKY, A.; BEN - DOR, E.; SAARONI, H, Diurnal thermal behavior of selected urban objects using remote sensing measurements. Energy and Buildings, n.36, p.1063 - 1074. 2004.

COSTA, M. H., and J. A. FOLEY.Water balance of the Amazon Basin: Dependence on vegetation cover and canopy conductance, J. Geophys. Res., 102(D20), 23,973-23,989, doi:10.1029/97JD01865, 1997.

COSTA, da M. L.; OLSZEVSKI. Caracterização da paisagem no Cerrado. In: Savanas desafios e estratégias para o equilíbrio entre sociedade, agronegócio e recursos naturais. Editores técnicos: Fábio Gelape Faleiro e Austeclinio Lopes de Farias Neto. EMBRAPA Cerrados. Planaltina, D.F., 2008.

FERREIRA JUNIOR, L. G. ; YOSHIOKA, H. ; HUETE, A. R. ; SANO, E. E. . Seasonal Landscape and Spectral Vegetation Index Dynamics in the Brazilian Cerrado: An Analysis within the Large Scale Biosphere-Atmosphere Experiment in Amazonia (LBA). Remote Sensing of Environment, Estados Unidos, v. 87, p. 534-550, 2003.

FERREIRA JUNIOR, L. G. ; HUETE, A. R. . Assessing the seasonal dynamics of the Brazilian Cerrado vegetation through the use of spectral vegetation indices. International Journal of Remote Sensing, Inglaterra, v. 25, n. 10, p. 1837-1860, 2004.

GOERT, J. W.; WAGNER, E.; BARCELLOS, O, de. A. Savanas tropicais: Dimensão, histórico e perspectiva. In: Savanas desafios e estratégias para o equilíbrio entre sociedade, agronegócio e recursos naturais. Editores técnicos: Fábio Gelape Faleiro e Austeclinio Lopes de Farias Neto. EMBRAPA Cerrados. Planaltina, D.F., 2008.

HASHIMOTO, H.; NEMANI, R.; YANG, F.; GRANGER, R.; RUNNING, S. VPD Estimation from Land Surface Temperature (MOD11) for global map of evapotranspiration and NPP. American Geophysical Union, Fall Meeting. 2003.

MARTINS JR., O. P. \& CHAVES, F. Uso de instrumentos econômicos para a conservação da biodiversidade em Goiás: reserva legal, servidão florestal e bolsa de reserva florestal. p. 165-175. In.: FERREIRA, L. G. (Org.) Encruzilhada ambiental, 2008.

MYERS, N.; MITTERMEYER, R. A.; MITERMEYER, C. G.; FONSECA, G. A.; KENT, J. Biodiversity hotspots for conservation priorities. Nature, 403, 853-858, 2000.

RATANA, P. ; HUETE, Alfredo Ramon ; FERREIRA JUNIOR, L. G. . Analysis of Cerrado physiognomies 
and conversion in the MODIS seasonal - temporal domain (LBA special issue). Earth Interaction, (In Press), v. 9, p. 1-22, 2005.

LEGENDRE, Pierre. Espatial autocorrelation: trouble or new paradigm? 4(6). pp 1659-1673, 1993.

RATER, J. A; BRIDGWATER, S.; ATKINSON, R.; RIBEIRO, J. F. Analysis of the florstic composition of the Brazilian Cerrado Vegetation II. Comparison of the woody vegetation of 98 areas. Edinburgh Jornal of Botanics, v. 53 (153 - 180). 1996.

NOBRE, A. CARLOS; SELLERS J. PIERS; SHUKLA. Amazonian deflorestation and regional climate change. Jornal of Climate, v.4, p.957 - 988, 1991.

PROBIO. Mapeamento da Cobertura Vegetal do Bioma Cerrado. Relatório Final, Brasília, 2007.

RIBEIRO. J, F.; WALTER. B, M, T, Fitofisionomias do bioma cerrado. In: Cerrado: ambiente e flora (S.M. Sano \& S.P. Almeida, eds). EMBRAPA-CPAC, p.89-166. Planaltina, 1998.

SANO, E. E.;ROSA, R.; BRITO J. L.; FERREIRA, L. G. Mapeamento semi-detalhado (escala de 1:250.000) da cobertura vegetal antrópica do bioma Cerrado. Pesquisa Agropecuária Brasileira, v. 43, n. 1 (153-156), 2008. SANO, E. E. ; ROSA, R.; BRITO, J. L. ; FERREIRA JR, L. G. Land cover mapping of the tropical savanna region in Brazil. Environmental Monitoring and Assessment, v. 000, p. 1-12, 2009.

SALISBURY, W.; D'ARIA, D.M, Emissivity of terrestrial material in the $8-14 \mathrm{~m}$ atmospheric window. Remote Sensing of Environment. 1992.

WAN, Z., Y.-Z,; FENG, Y,; ZHANG,; KING. M. D. Land-surface temperature and emissivity retrieval from MODIS Airborne Simulator (MAS) data, Summaries of the Seventh JPL Airborne Earth Science Workshop, January 12-16,vol. 3, pp.57-66. 1998.

WAN. Z. New refinements and validation of the MODIS land-surface temperature/emissivity products , International Journal of Remote Sens. Environ., in press. 2007a.

WAN, Z. MODIS Land Surface Temperature Products Users' Guide Collection-5. ICESS, University of California, Santa Barbara, 2007b.

Trabalho enviado em janeiro de 2011 Trabalho aceito em abril de 2011 
SANTOS, N. B. F.; FERREIRA JÚNIOR, L. G.; FERREIRA, N. C. 